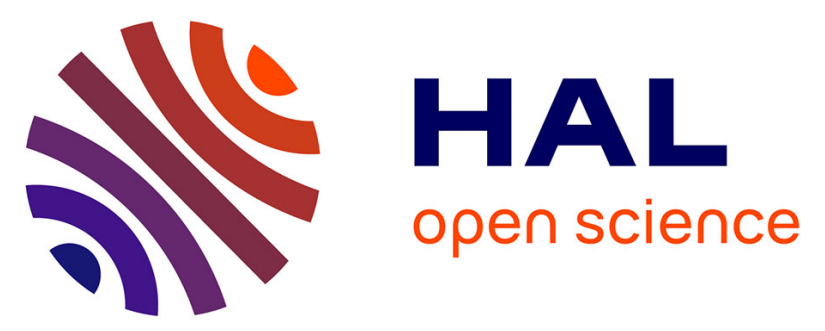

\title{
Histoire des lettres et tradition de l'erreur. De l'Avis pour dresser une bibliothèque de Naudé au Dictionnaire historique et critique de Bayle
}

Bernard Teyssandier

\section{- To cite this version:}

Bernard Teyssandier. Histoire des lettres et tradition de l'erreur. De l'Avis pour dresser une bibliothèque de Naudé au Dictionnaire historique et critique de Bayle. Littératures classiques, 2015, Naissance de la critique littéraire, 86 (1), pp.67-94. 10.3917/licla1.086.0067 · hal-02078078

\section{HAL Id: hal-02078078 \\ https://hal.univ-reims.fr/hal-02078078}

Submitted on 6 Jan 2022

HAL is a multi-disciplinary open access archive for the deposit and dissemination of scientific research documents, whether they are published or not. The documents may come from teaching and research institutions in France or abroad, or from public or private research centers.
L'archive ouverte pluridisciplinaire HAL, est destinée au dépôt et à la diffusion de documents scientifiques de niveau recherche, publiés ou non, émanant des établissements d'enseignement et de recherche français ou étrangers, des laboratoires publics ou privés. 


\title{
Histoire des lettres et tradition de l'erreur: de l'Avis pour dresser une bibliothèque au Dictionnaire historique et critique
}

\author{
Bernard TEYSSANDIER \\ Université de Reims Champagne-Ardenne
}

À Julie Boch ${ }^{1}$ (1969-2011)

Dans un texte paru en 1843 dans La Revue des deux mondes, Sainte-Beuve, conservateur à la Mazarine, imagine de déplacer le buste de Gabriel Naudé pour le disposer dans «son vrai lieu », c'est-à-dire «entre Charron, ou mieux, entre Montaigne et Bayle ${ }^{2}$ ». $\mathrm{Au}$ delà de l'anecdote, cette révision taxinomique témoigne d'une juste et large vue sur l'histoire de la pensée à l'âge classique, comme le confirment les dernières lignes de l'Avis pour dresser une bibliothèque. Le jeune et ambitieux Naudé qui dédie son traité à son nouveau patron, le Président Henri de Mesmes, conclut ainsi son propos :

Comme j'ai pris dès maintenant la hardiesse de vous [...] donner, par cet Avis, [la preuve de mon engagement à augmenter votre bibliothèque], j'espère bien, avec le temps, polir et augmenter [ce même Avis], de telle sorte qu'il n'appréhendera point de sortir en lumière pour discourir et parler amplement d'un sujet lequel n'a point encore été traité, faisant voir sous le titre de Bibliotheca Memmiana ce qu'il y a si longtemps que l'on souhaite savoir, l'histoire très ample et particulière des lettres et des livres, le jugement et censure des auteurs, le nom des meilleurs et plus nécessaires en chaque faculté, le fléau des plagiaires, le progrès des sciences, la diversité des sectes [c'est-à-dire des écoles de pensées], la révolution des arts et disciplines, la décadence des anciens, les divers principes des novateurs, et le bon droit des Pyrrhoniens fondé sur l'ignorance de tous les hommes. ${ }^{3}$

Au vu d'un tel programme, où le projet annoncé d'une historia litteraria s'accompagne d'une adhésion à la sagesse des Pyrrhoniens, comment ne pas penser à Montaigne en effet, pour qui la lecture des livres ne devait et ne pouvait être une simple «opération d'enregistrement ${ }^{4}$ »? Le rattachement au scepticisme procède bien chez Naudé comme chez l'auteur des Essais d'une tradition philosophique qui voit dans le temps consacré aux études et dans la lecture des bons auteurs non pas tant «une puissance d'accumulation et de sédimentation » qu' «une force d'érosion » à même de saper « toute prétention humaine à la fixation d'une vérité » en ramenant «sans fin l'effort de connaissance au néant de l'ignorance ${ }^{5}$ ». Quant à l'idée d'une histoire des savoirs fondée sur le principe d'un rassemblement des connaissances et d'une évaluation des textes à l'aune de la raison, elle consonne très largement aussi avec le projet d'un dictionnaire dont la dimension à la fois historique et critique trouverait dans la philosophie sceptique sa justification et sa régulation - l'accumulation des livres allant de pair avec leur relativisation.

\footnotetext{
${ }^{1}$ Pierre Bayle, Pensées sur l'athéisme, éd. par J. Boch, Paris, Desjonquères, 2004.

${ }^{2}$ Sainte-Beuve, Gabriel Naudé, Paris, Paris-Zanzibar, 1999, p. 19.

${ }^{3}$ Avis pour dresser une bibliothèque, éd. par Bernard Teyssandier, Paris, Klincksieck, 2008, p. 339 et 341.

${ }^{4}$ Jean-Marc Chatelain, La Bibliothèque de l'honnête homme. Livres, lecture et collection en France à l'âge classique, Paris, Bibliothèque nationale de France, 2003, p. 16.

${ }^{5}$ J.-M. Chatelain et B. Teyssandier (dir.), «L'idée des bibliothèques à l'âge classique », Littératures classiques, 66, 2008, «présentation », p. 10.
} 
Pour autant, 1'hypothèse avancée naguère par Jean-Marc Chatelain et moi-même selon laquelle le Dictionnaire de Bayle constituerait sinon l'application des préceptes allégués par Naudé soixante-dix ans plus tôt dans son Avis, du moins la réalisation annoncée par lui et jamais advenue de cette Bibliotheca Memmiana promise à son mécène dédicataire, cette hypothèse est-elle soutenable ${ }^{6}$ ? Pour le dire autrement, le Dictionnaire historique et critique, dont les deux éditions parues du vivant de son auteur datent respectivement de 1697 et 1702 , peut-il vraiment être évalué à partir du projet d'historia litteraria que le « libertin érudit ${ }^{7}$ » se promettait de réaliser dans le prolongement logique de son Avis pour dresser une bibliothèque?

\section{I- «Progrès et augmentation du savoir » : l'idée de bibliothèque de Gabriel Naudé}

Conformément aux espoirs et même aux prédictions du jeune Naudé qui, dès les premières lignes de son discours, se présente sous les traits d'un athlète de la pensée doublé d'un précurseur du savoir - «je me suis, le premier, efforcé de rompre la glace et tracer le chemin en courant à ceux qui le voudront rebattre plus à loisir ${ }^{8} »-$, L'Avis pour dresser une bibliothèque fit date dans l'histoire. Pour la première fois, l'économie de la bibliothèque y était envisagée dans sa globalité. En neuf chapitres serrés, Naudé exposait son dessein en s'attachant au moyen de "recouvrer » les livres, c'est-à-dire de les rechercher, au lieu le mieux adapté établir la collection et assurer sa conservation, à l'ordre à adopter pour ranger les ouvrages, enfin, et presque à contrecœur tant la question lui semblait secondaire, aux éléments constitutifs de la décoration. Discours ambitieux, certes, surtout au vu du colossal chapitre quatre, massif de près de soixante pages pour un livre qui n'en compte guère plus de cent soixante, destiné à aborder la question du choix des livres et de leurs auteurs.

Dans sa prétention à embrasser tous les savoirs du monde, Naudé hérite de l'idée d'encyclopédie telle qu' elle fut théorisée à la Renaissance. Son projet est d'inspiration ramiste en cela qu'il envisage la bibliothèque comme une totalité cohérente relevant d'un processus complexe par spécifications successives ${ }^{9}$. Mais Naudé est aussi redevable à la philosophie de Francis Bacon, et notamment à l'ouvrage paru à Londres en 1605 sous le titre de Proficience and Advancement of Learning dans lequel l'auteur infléchit la proposition d'un savoir organisé dont la nature relèverait d'un système par prolifération et subdivisions, en lui associant l'idée d'expansion renouvelée, c'est-à-dire de progression ${ }^{10}$. Dans l'épigramme qu'il écrit à l'occasion de la réédition du texte de Naudé en 1644, Jean-Cécile Frey, grand diffuseur de la pensée de Pierre de la Ramée en France et ancien professeur de Naudé, reprend d'ailleurs à son compte la métaphore baconienne d'une avancée du savoir pour l'appliquer à la bibliothèque telle que la pense Naudé :

Car, si une plante est incapable de générer une autre plante,

\footnotetext{
${ }^{6}$ « D'où l'on pourrait conclure que l'idée naudéenne de la bibliothèque trouvera sa réalisation la plus accomplie en 1697 dans le Dictionnaire de Bayle, justement, dit historique et critique », J.-M. Chatelain et B. Teyssandier (dir.), Littératures classiques, 66, 2008, «présentation », p. 10.

${ }^{7}$ René Pintard, Le Libertinage érudit dans la première moitié du XVII siècle (1943), Genève, Slatkine reprints, 2000.

${ }^{8}$ Avis pour dresser une bibliothèque, op. cit., p. 7.

9 Jean Lecointe, L'Idéal et la Différence. La perception de la personnalité littéraire à la Renaissance, Genève, Droz, 1993, p. 17.

${ }^{10}$ Voir l'avant-propos que Michèle Le Dœuff consacre au livre et à sa réception en France au XVII ${ }^{\mathrm{e}}$ siècle : Francis Bacon, Du progrès et de la promotion des savoirs (1605), Paris, Gallimard, 1991, p. VII-LXIV. Voir aussi notre compte rendu : Estelle Bœuf, La Bibliothèque parisienne de Gabriel Naudé en 1630, Genève, Droz, 2007 : Bulletin du bibliophile, 2008, $\mathrm{n}^{\circ}$ 1, p. 199-203.
} 
Une bibliothèque, elle, peut en générer une autre. ${ }^{11}$

L'idée qui prévaut, sous-tendue par l'image de l'arbre, sans doute empruntée au néoplatonicien Porphyre ${ }^{12}$, n'est plus exactement celle d'une totalité dont dépendraient les parties adjacentes, mais bien celle d'un développement par irrigation et génération où la totalité s'estomperait au profit de l'avancée ${ }^{13}$.

Cette image énergique est très largement relayée par Naudé dans son traité, par exemple dans l'épître liminaire adressée à Henri de Mesmes :

Si vous ambitionnez de faire éclater votre nom par celui de votre bibliothèque $[\ldots]$ il est besoin d'augmenter et de perfectionner tous les jours ce que vous avez si bien commencé et donner insensiblement un tel et si avantageux progrès à votre bibliothèque. ${ }^{14}$

Or quelle est cette force qui garantit progrès, augmentation et promotion à la bibliothèque, quelle est cette sève qui assure non seulement l'irrigation mais aussi la conservation d'une collection apte à se régénérer ? Sans doute une pensée dominée par la raison autonome et, de ce fait, rendue suffisamment forte pour éradiquer les fausses croyances, repousser les chaînes de l'opinion et diriger le jugement. Autrement dit une forme d'esprit critique que Naudé, dans tous les textes qui précèdent l'Avis, qu'il s'agisse du Marfore (1620), de l'Instruction à la France sur la vérité de l'histoire des Frères de la Rose-Croix (1623) ou encore de l'Apologie pour tous les grands personnages qui ont été faussement soupçonnés de magie (1625), exerce en préalable à toute appréciation et interprétation.

Dans sa capacité à identifier les forces hostiles au progrès de la connaissance et à les combattre, Naudé demeure une fois encore redevable à la pensée de Bacon. Lorsqu'il considère, par exemple, qu'il n'appartient qu'aux esprits supérieurs « de se délivrer de la servitude et esclavage de certaines opinions qui nous font régler et parler de toutes choses à notre fantaisie et de juger à propos et sans passion du mérite et de la qualité des auteurs ${ }^{15}$ », il désigne par d'autres mots ce que Bacon appelle pour sa part les «idoles [...] inhérentes à la tribu $^{16}$ ». Pour Naudé simplement, ces ennemis se nomment «admiration», esprit d'enthousiasme, esprit de parti, en somme parti pris.

Mais contrairement à Bacon qui pense la progression du savoir à partir du Livre de Dieu et de la Nature pour ne se cantonner qu'au second ${ }^{17}$, Naudé construit sa bibliothèque en les réunissant. Bien entendu, il se garde d'attaquer de front l'orthodoxie ; tout juste s'emploie$\mathrm{t}$-il à contester son bien-fondé, et encore de manière à n'être compris que d'un petit nombre ${ }^{18}$. À y bien regarder pourtant, Naudé sape les prétentions de la doxa dans son Avis : en associant d'abord l'ordre de la foi et celui de la raison, et en amalgamant ensuite à l'idée baconienne du progrès celle, d'esprit plus libertin et en tout cas plus pyrrhonien, de « révolution » et de « décadence ».

Arrêtons-nous sur ces deux points. Le premier d'abord. L'Avis pour dresser une bibliothèque pouvait-il décemment penser l'universalité des savoirs sans en référer à l'ordre

\footnotetext{
${ }^{11}$ Avis pour dresser une bibliothèque, op. cit., p. 11, note b.

${ }^{12}$ J. Lecointe, op.cit., p. 27.

${ }^{13}$ J.-M. Chatelain, "Du Parnasse à l'Amérique : l'imaginaire de l'encyclopédie à la Renaissance et à l'Âge classique ", dans Tous les savoirs du monde, Encyclopédies et bibliothèques, de Sumer au XXI siècle, Roland Schaer (dir.), Paris, Bibliothèque nationale de France/ Flammarion, 1996, p. 156-163.

${ }_{14}^{14}$ Avis pour dresser une bibliothèque, op. cit., p. 27 et 29.

${ }^{15}$ Ibid., p. 19 et 21.

${ }^{16}$ M. Le Dœuff, texte cité, p. XXXII.

${ }^{17}$ Ibid., p. LII-LIII.

${ }^{18}$ B. Teyssandier, «L'ethos érudit dans l'Avis pour dresser une bibliothèque de Gabriel Naudé », Littératures classiques, 66, 2008, p. 129-130.
} 
de la foi ? Évidemment non. Le Livre de Dieu trouve non seulement place dans la bibliothèque universelle mais il occupe même apparemment la toute première place : dans le chapitre qu'il consacre à la qualité des livres, Naudé dispose les « diverses bibles des Pères et des conciles» et «le gros de la théologie» en tête du cortège, comme si le statut dont jouissaient ces textes dans l'économie des savoirs institués leur conférait naturellement une autorité. L'auteur de l'Avis va même plus loin puisqu'il profite de l'occasion pour dire publiquement son allégeance à l'Église souveraine, se payant même le luxe d'affirmer que le Talmud et l'Alcoran, "vomissent mille blasphèmes contre Jésus-Christ et notre religion ${ }^{19}$ ", ce qui ne l'empêche pas par ailleurs de les accueillir dans sa bibliothèque...

La manœuvre est habile en réalité : en associant tous les livres, et le plus grand nombre de livres même, ceux de Dieu et ceux de la Nature, en " enchaînant » les auteurs les uns aux autres, Naudé rend possible leur confrontation et de fait prépare leur relativisation :

[...] en théologie, par exemple, il faut mettre toujours les Bibles les premières, suivant l'ordre des langues, par après les conciles, synodes, décrets, canons, et tout ce qui est des constitutions de l'Église, d'autant qu'elles tiennent le second lien d'autorité parmi nous; ensuite, les Pères grecs et latins et, après eux, les commentateurs, scolastiques, docteurs mêlés, historiens et, finalement, les hérétiques. [...] et, finalement, tous [ces auteurs] qui se sont exercés en pareille escrime, et qui sont tellement enchaînés les uns avec les autres, qu'il y aurait autant de faute à les lire séparément comme à juger et entendre une partie sans l'autre, ou un contraire sans celui qui lui est opposé. ${ }^{20}$

Dans la mesure où la bibliothèque universelle a vocation agonistique, la critique, d'une certaine manière, lui est consubstantielle.

Venons-en à présent au second point qui procède de l'intégration de l'idée de révolution et de décadence à celle, d'origine baconienne, de progrès des sciences. Naudé ne se contente pas de théoriser la bibliothèque universelle, il dresse aussi le bilan du savoir humain. Or que constate-t-il ? Que la connaissance est constamment soumise à l'évaluation. Et ce qui s'est produit pour les livres de la Nature n'augure rien de bon, sans doute, pour les livres de Dieu. En réunissant théologie et philosophie, Naudé soumet ces deux savoirs aux lois de l'évolution c'est-à-dire de la destruction - nouvelle preuve du vrai triomphe des Pyrrhoniens dont l'objectif est bien « de renverser toutes les sciences ${ }^{21} »$.

On comprend ici les raisons qui conduisent l'auteur de l'Avis pour dresser une bibliothèque à contredire Sénèque, qui n'en constitue pas moins sa principale référence en matière de morale, sur l'épineuse question de l'excès des livres et du savoir bibliographique ${ }^{22}$. Si Naudé n'a de cesse de faire entrer un très grand nombre de livres dans sa bibliothèque, c'est parce que ceux-là même qui véhiculent les mensonges les plus dangereux et les plus vils, seront justement, dans l'enceinte d'un lieu créé pour les recevoir, disponibles pour être critiqués :

Car, encore bien que la plupart [des matières semblables à la pierre philosophale, divinations] n'enseignent rien que des choses vaines et inutiles, [...] si est-ce néanmoins que, pour avoir de quoi contenter les faibles esprits aussi bien que les forts, et satisfaire au moins à ceux qui les

\footnotetext{
${ }^{19}$ Avis pour dresser une bibliothèque, op. cit., p. 91 et 121.

${ }^{20}$ Ibid., p. 277 , p. 103 et p. 105.

${ }^{21}$ Ibid., p. 103.

22 J.-M. Chatelain, «L'excès des livres et le savoir bibliographique », Littératures classiques, 66, 2008, p. 145160.
} 
veulent voir pour les réfuter, il faut recueillir ceux qui en traitent, dussent-ils être, parmi les autres livres d'une bibliothèque, comme les serpents et vipères entre les autres animaux, comme l'ivraie et le bon grain, comme les épines entre les roses, et ce, à l'exemple du monde où ces choses inutiles et dangereuses accomplissent le chef-d'œuvre et la fabrique de sa composition. ${ }^{23}$

Le jugement sur les mauvais livres devient ainsi la condition nécessaire à l'avènement de la vérité des livres.

Mais s'il convient d' ' ouvrir » la bibliothèque à un grand nombre d'ouvrages, il faut aussi, et dans le même temps, veiller à préserver l'émulation qui préside à l'avancée du savoir. Dans ces conditions, il est plus que nécessaire de faire barrage à ce que Naudé appelle les «livres rampants ${ }^{24}$ » désignant par là à la fois les auteurs modernes «coiffés et embéguinés de tous les nouveaux livres ${ }^{25}$ », les « rhapsodeurs, copistes ou plagiaires ${ }^{26}$ » et les thuriféraires du passé qui se «tiennent aussi fermement attachés [aux anciens] comme le poulpe fait à la roche ${ }^{27}$ ». Pour l'auteur de l'Avis pour dresser une bibliothèque, en effet, la vipère n'a de bon que si elle pique. Dès lors, la condamnation des faux livres et non pas des mauvais va de pair chez Naudé avec le mépris manifesté à l'encontre des collectionneurs frileux dont l'objectif principal relèverait d'un «contentement particulier ${ }^{28}$ » et pour lesquels la « librairie » ne constituerait qu'un lieu fermé. Parce que l'idée de bibliothèque universelle est incompatible avec le vice de " philautie ${ }^{29}$ », toute tentative visant à confisquer le savoir au profit d'un seul est un frein à l'avancée des connaissances : par le truchement des livres et des bons auteurs, le lecteur devra au contraire s'éprouver comme une conscience individuelle engagée dans une histoire collective, comme un être singulier dépendant d'une communauté ${ }^{30}$.

\section{II- Histoire héroïque et critique historique : Moreri et Bayle}

Grand lecteur lui-même et non moins grand compilateur, Bayle, se range comme Naudé à la sagesse des sceptiques: son Dictionnaire s'attache à déceler l'erreur dans les livres et s'inscrit moins dans une démarche édificatrice visant à faire éclater la vérité que dans la capacité de la raison à pouvoir déceler l'erreur. Pour mener à bien cette lourde tâche, Bayle choisit le dictionnaire : comme support d'écriture, d'abord, ce qui lui permet, notamment grâce aux notes, de démontrer qu'il y a bien eu erreur, comme objet de lecture ensuite, les dictionnaires, sortes de bibliothèques portatives, lui semblant d'une grande commodité pour déceler ces fautes, les examiner plus aisément et en évaluer la portée.

Or parmi les nombreux calepins du XVII ${ }^{\mathrm{e}}$ siècle, il en est un qui, aux yeux de Bayle, frappe par le nombre d'erreurs qu'il recèle justement, et il s'agit du Moreri. Paru en un volume au format in-folio en 1674, augmenté d'un second tome en 1681, un an tout juste après de la mort de son auteur, Le Grand dictionnaire historique ou le mélange curieux de l'histoire sainte et profane, rapportant en abrégé, les Vies des patriarches, Juges et rois de l'Ancien Testament, des Souverains pontifes de l'Eglise, des saints Pères et docteurs

\footnotetext{
${ }^{23}$ Avis pour dresser une bibliothèque, op. cit., p. 115 et 117.

${ }^{24}$ Ibid., p. 133.

${ }^{25}$ Ibid., p. 171.

${ }^{26}$ Ibid., p. 147.

${ }^{27}$ Ibid., p. 149.

${ }^{28}$ Ibid., p. 25.

${ }^{29}$ Ibid., p. 21.

30 «L'ethos érudit dans l'Avis pour dresser une bibliothèque de Gabriel Naudé », art. cit., p. 117-131.
} 
orthodoxes, des évêques des quatre églises patriarcales, des cardinaux et prélats célèbres et des hérésiarques. Celles des Empereurs de Rome, de Grèce, d'Allemagne, païens, chrétiens et Ottomans, des rois, des princes illustres, et des grands capitaines, des auteurs grecs et latins, anciens et modernes, des philosophes, des inventeurs des arts [...] contenant : la description des Etats, Empires, Royaumes, Provinces, Villes, Iles, Montagnes et Fleuves considérables de l'ancienne et nouvelle géographie, où l'on remarque exactement les bornes, la situation et les qualités des pays, les mours, les coutumes, le gouvernement et la religion des peuples. Avec l'histoire des Conciles généraux et particuliers, synodes, conciliabules, et autres assemblées ecclésiastiques, en parlant des villes où elles ont été tenues. Le nom, l'établissement et la propagation des ordres religieux, et militaires, et la vie de leurs fondateurs. Et l'histoire fabuleuse des dieux et héros de l'Antiquité païenne. Le tout enrichi de remarques et dissertations curieuses, tant pour l'éclaircissement des difficultés de chronologie que pour la décision des controverses historiques aspire bien, comme son titre interminable l'annonce, à la totalité.

Mais quels sont les critères qui sous-tendent exactement cette démarche universaliste ? En réalité l'ouvrage entend faire non pas l'histoire du savoir mais celle des personnes renommées pour leur « érudition » ou pour leurs « actions éclatantes ${ }^{31}$ ». Aussi ce dictionnaire est-il surtout " grand » par le fait qu'il réunit, en un vaste bouquet, les plus belles fleurs de l'histoire humaine. L'ouvrage procède ainsi d'une éthique et d'une esthétique de la gloire. La formule qui sert de clausule à la première version est d'ailleurs révélatrice-Merces mihi gloria detur : « que j'aie la gloire pour fruit de mon travail ». Moreri, qui s'est évertué à faire entrer les hommes illustres dans son Grand dictionnaire aspire à son tour, par son dictionnaire justement, à la postérité.

Cette vision d'une Histoire tout entière façonnée par les individus et cette posture d'écrivain grand dispensateur de médailles trouve naturellement sa traduction rhétorique dans l'adoption en continue du genre épidictique : les entrées du Grand Dictionnaire prodiguent tour à tour des éloges ou des blâmes, mais des éloges surtout, le commentaire n'étant que très rarement polémique - « je n'ai le dessein d'offenser personne ${ }^{32}{ }$ ' indique prudemment Moreri en avant-propos.

Pour autant, et quoi qu'il en ait, Moreri n'est pas la voix de l'Histoire, tout au plus prête-t-il la sienne à la doxa. Érudit et prêtre, il confesse à la fin de son épître dédicatoire que « chrétien » est son nom et « catholique » son surnom :

[...] il est sûr que je n'ai rien écrit contre ma conscience ni contre la vérité qui m'ait été connue. Aussi je me soumets au jugement d'un équitable lecteur [...]. Je soumets aussi cet ouvrage au jugement de l'Église catholique, apostolique et romaine, que je reconnais pour ma bonne et unique mère et pour ma maîtresse. ${ }^{33}$

L'entrée qu'il consacre à Louis XIV en 1674 prouve encore sa totale fidélité au pouvoir monarchique ${ }^{34}$. Quant à Parayre, il marche sur les brisées du maitre en dédiant au roi de France le Dictionnaire de Moreri dont il réalise l'augmentation en 1681 :

[Louis Moreri] a enfin considéré V.M. comme un roi qui a rassemblé en sa personne le mérite de tous ces grands princes dont les siècles passés ont célébré la mémoire. C'est dans ce sentiment, SIRE, que cet excellent auteur

\footnotetext{
${ }^{31}$ Le Grand dictionnaire historique ou le mélange curieux de l'histoire sacrée par le Sieur L. Moreri, Lyon, Jean Girin, Barthélemy Rivière, 1674, «préface », texte non paginé.

${ }^{32}$ Ibid.

${ }^{33} \mathrm{Ibid}$.

${ }^{34}$ Ibid., p. 817-818.
} 
aurait voulu nous laisser l'histoire entière de VOTRE MAJESTE, mais connaissant bien qu'il n'est pas moins difficile d'écrire tous ses miracles, qu'il est impossible de les imiter, il s'est contenté de nous représenter en général l'autorité que VM a si heureusement employé pour abattre l'hérésie, pour conserver la pureté de la religion, et pour étouffer toutes les semences de nouveauté, le bon ordre qu'elle a rétabli dans l'administration de la justice, et des finances, la rigueur qu'Elle a exercée pour arrêter la fureur des duels, et particulièrement sa conduite si admirable en ses armées toujours victorieuses qui ont rendu VM l'arbitre de la destinée de toute l'Europe. ${ }^{35}$

Pour Moreri et Parayre, sans doute, Le Grand dictionnaire historique atteint la « perfection ${ }^{36} »$ tant il est vrai qu'il vise à l'exhaustivité tout en contribuant à l'exemplarité.

À cette logique de la monumentalité répond de manière assez amusante, il faut en convenir, tant la dissonance est frappante, le «projet» de Bayle, texte publié cinq ans avant que ne paraisse son propre Dictionnaire, et dans lequel l'auteur parle d' "ébauche », $\mathrm{d}^{\prime}$ ' essai $^{37}$ » et évoque mi-goguenard mi-sérieux la tradition savante du theatrum. Le nouveau " théâtre » dont il est l'inventeur, assure Bayle, ne sera pas moins "divertissant et moins instructif que celui de la vie humaine » de Theodor Zwinger (1565), simplement, au lieu d'offrir aux spectateurs le spectacle des vérités humaines il lui montrera ses très nombreuses « fautes ».

Quand Moreri superpose ses textes de manière linéaire dans un effet qui relève du feuilletage, Bayle dispose ses entrées en deux parties, l'une horizontale, où des faits synthétiques sont allégués, l'autre verticale, souvent beaucoup plus développée, l'auteur examinant, citations à l'appui, et grâce au commentaire, les allégations antérieures pour les valider, les infléchir ou les contester. À une parole prophétique et assertive répond ainsi une démarche évaluative et démonstrative portée par une langue ondoyante. À une juxtaposition textuelle des effets de croisements, de renvois, de redoublement - la note est censée faire écho parfois à d'autres notes, notes marginales de nature bibliographiques le plus souvent, ou notes d'autres entrées du dictionnaire. Bref, la matérialité de l'ouvrage signale qu'on a quitté l'espace du prestige livresque pour celui du bric-à-brac, agrégat textuel dont l'ordre et la logique ne répondent plus aux lois canoniques de la belle mise en page. À l'héroïsme revendiqué de Moreri répond ainsi une sorte de prosaïsme assumé : dans «le projet d'un dictionnaire critique », Bayle va même jusqu'à annoncer son intention de rendre publique la « chambre d'assurance de la République des lettres » et d'exhiber ses « ramas [d']ordures ». Autant dire que la parole encomiastique n'a plus sa place dans ce calepin d'esprit picaresque : « on prend une route », avertit son auteur, « qui n'est pas celle de la louange ${ }^{38} »$.

En réalité, il s'agit bien ici comme préalablement chez Naudé, d' « apprendre à ne pas croire légèrement ce qui s'exprime ${ }^{39}$ » et ce qui s'imprime en se débarrassant des «idoles » que sont la mauvaise foi, l'éblouissement ou simplement la petitesse d'esprit. On comprend, dans ces conditions, que le Grand Dictionnaire de Moreri soit décrié :

\footnotetext{
${ }^{35}$ Le Grand dictionnaire historique ou le mélange curieux de l'histoire sacrée, augmenté et divisé en deux tomes, seconde édition, Lyon, Jean Girin et Barthélemy Rivière, 1681, 2 vol., t. I, « Au roi », texte non paginé.

${ }^{37}$ « Je me suis mis en tête de compiler le plus gros recueil qu'il me sera possible de fautes dans les dictionnaires, et de ne pas me renfermer dans ces espaces, quelques vastes qu'ils soient, mais de faire aussi des courses sur toutes sortes d'auteurs, quand l'occasion s'en présentera. [...] je ne me propose que de [...] fournir [aux savants] un essai, ou une ébauche qui puisse en déterminer quelques-uns à perfectionner ce plan et à grossir de plusieurs volumes ce dictionnaire critique » : «Projet d'un dictionnaire critique, à Mr du Rondel, professeur aux BellesLettres à Maastricht », dans Projet et fragments d'un dictionnaire critique, Rotterdam, Reiner Leers, 1692, texte non paginé.

${ }^{38}$ Ibid.

${ }^{39}$ Ibid.
} 
Il n'y a rien de plus ridicule qu'un dictionnaire où l'on fait le controversiste.

C'est un des plus grands défauts de celui de Mr Moreri, on y trouve cent endroits qui semblent être détachés d'un vrai sermon de croisade. ${ }^{40}$

Pour Bayle en effet la raison trouve sa justification dans le fait qu'elle la plus à même de déceler l'erreur et à détecter le mensonge :

J'entends par mentir [...] non seulement l'invention entière d'un fait faux, mais aussi la suppression ou l'addition de certaines circonstances qui peuvent servir ou à disculper les gens, ou à les charger. ${ }^{41}$

Contrairement au Grand dictionnaire, le Dictionnaire historique et critique de Bayle n'aspire pas à la «perfection ». Pour Bayle, d'ailleurs, la perfection n'est pas de ce monde, les avancées de la raison critique demeurant sujettes à constantes réévaluations :

[...] c'est le fort des dictionnaires de ne marcher vers la perfection que fort lentement, et qu'à diverses reprises. Il leur manque une infinité de choses la première fois qu'ils se montrent, le temps les leur donne peu à peu. ${ }^{42}$

Ces différences de fond, ces divergences d'appréciations entre le Dictionnaire de Bayle et celui de Moreri se retrouvent dans les images qui figurent au seuil de ces deux massifs d'articles.

Livre monumental au format in-folio, le Dictionnaire historique de Moreri adopte naturellement la forme du titre-frontispice à pleine page. La gravure sur cuivre, signée de Jean Audran, est d'inspiration héroïque : décor à l'antique, fûts cannelés coiffés de chapiteaux corinthiens, colonnes ornées de feuilles de lauriers torsadées, trophées. Le paysage qui se profile en point de fuite consonne avec ces représentations : l'obélisque, les statues en pieds qui coiffent les toits des palais évoquent l'idée d'un triomphe éternel dans le cadre d'une ville symbolisant elle-même l'éternité : la Rome tridentine [FIGURE 1]. L'image évoque à la fois la collection et le trésor : collection et trésor que l'œuvre de Moreri ramasse, organise et exhibe.

Au premier plan de cette image, l'Histoire est représentée une plume à la main, tenant un livre ouvert - Le Grand Dictionnaire historique bien entendu. Cette allégorie est très logiquement accompagnée du Temps pour signifier que la matière historique a vocation à embrasser le savoir humain dans sa totalité, eu égard notamment aux louanges ou aux blâmes décernés par la postérité. Mais ce qui est le plus intéressant dans cette composition, c'est que les personnages du premier plan indiquent tous d'un geste de la main une même direction. Le spectateur est ainsi invité à porter ses regards sur la partie gauche de l'image, et plus exactement sur le tableau enchâssé dans l'ample piédestal coiffant les colonnes ornées [FIGURE 2]. Par ce geste impérieux, l'Histoire signale que son propre enseignement repose sur la représentation de l'Histoire. Et d'ailleurs l'Histoire est elle-même en représentation ici : sur le tableau enchâssé figurent des insignes du pouvoir temporel et spirituel - tiares, mitres, couronnes et foudres. On a là figurées l'alliance de la monarchie et de l'Église catholique, association du spirituel et du politique - idéal qu'un protestant comme Bayle ne pouvait, soit dit en passant, que réprouver.

\footnotetext{
40 Ibid.

${ }^{41}$ Article Rémond rem. D. : texte cité par Elisabeth Labrousse, dans Notes sur Bayle, Paris, Jean Vrin, 1987, «La méthode critique chez Pierre Bayle et l'Histoire », p. 454.

${ }^{42}$ Dictionnaire historique et critique par Monsieur Bayle, éd. 1702, Rotterdam, Reiner Leers, 3 vol., t. I, «Avertissement sur la seconde édition », texte daté du 7 décembre 1701, non paginé.
} 
Mais ce qui contrevient surtout à l'esprit du Dictionnaire de Bayle, c'est ce que suggère le décor figuré au-dessus de l'allégorie du Temps. Dans une niche figure un bouquet de fleurs, ample bouquet qui occupe tout l'espace du creux dans lequel il est disposé, et que l'inscription épigraphique qui court sur l'arrondi de la niche, invite à interpréter symboliquement. Cette expression latine, Ex pluribus unus odor signifie littéralement «Une seule odeur extraite de toutes ». [FIGURE 3] L'idée repose sur l'alliance du rassemblement le bouquet - et de la variété réunifiée : une seule odeur. C'est bien de fait la tradition, tradition politique et religieuse, qui fait ici l'objet d'une vénération : tous les personnages du premier plan la contemplent et la méditent. La Grande Histoire se confond ainsi avec l'histoire de l'orthodoxie.

La gravure de titre du Dictionnaire de Bayle dessinée par Adrian van der Werff sera le plus souvent reprise dans les éditions du XVIII siècle. [FIGURE 4] Les effets d'économie et de simplicité - médaillon gravé et non plus titre frontispice, décor d'encadrement en bois d'épines et non pas, comme il était d'usage, en branches de laurier tressées - traduisent une certaine inflexion : on n'est plus stricto sensu dans une logique de la splendeur même si le décor à l'antique conserve à l'ensemble une forme de majesté. Mais la solennité repose ici essentiellement sur la sobriété. Qui plus est, ce n'est pas 1'Histoire qui est représentée au premier plan mais la Sagesse. Le détail est d'importance : c'est Minerve, l'allégorie de la Raison, qui lit l'Histoire, laquelle perd son statut de sujet enseignant pour devenir objet d'enseignement.

Or sur quoi repose cet apprentissage ? Non plus sur la mise en spectacle et sur la mise en image mais sur la lecture. La grande quantité de livres ouverts, empilés les uns sur les autres témoigne de lectures redoublées et successives. Minerve désigne d'ailleurs du doigt non plus une image, comme l'Histoire le faisait dans la gravure de Jean Audran, mais un texte. Bref, on a bien la représentation d'une méthode critique reposant sur la vérification. L'artiste cherche à traduire, par le dessin, l'idée d'une activité intellectuelle reposant sur l'ordre des livres - traduction possible d'une recherche historique collective que Bayle appelle d'ailleurs de ses vœux dans son projet et dans la préface de son Dictionnaire. Le livre que Minerve tient ouvert est non seulement ouvert mais il est lu par elle, contrairement à celui que l'Histoire dans le frontispice du Moreri exhibait à la vue du spectateur. Ici, la discussion pourrait même porter sur le passage précis d'un texte, celui que Minerve indique de la main gauche sur la page du livre reposant sur ses genoux. Les faits allégués par ce passage sont ensuite examinés : Minerve en évalue la validité en les confrontant à un nouveau passage qu'elle indique de la main droite sur l'ouvrage que le putto, debout à côté d'elle, tient ouvert pour lui en faciliter l'accès, tandis que ses deux autres collaborateurs, les putti assis à ses pieds, accomplissent d'autres vérifications - les corps de ces lecteurs penchés vers l'objet de leur quête pouvant même souligner l'engagement et l'investissement personnels que l'un et l'autre portent à cette recherche. Quoi qu'il en soit, c'est la raison en travail qui est ici représentée, ce n'est plus l'Histoire en majesté - Minerve d'ailleurs, conformément à l'usage, est casquée, et non plus laurée, signifiant par là que la vérité se conquiert davantage qu'elle ne se contemple. [FIGURE 5]

\section{III- Enjeux et avatars de l'ars critica}

Pour Naudé comme pour Bayle l'histoire du savoir est indissociable d'une tradition de l'erreur que seule une méthode dictée par la raison, nommée faute de mieux critique, est à même de découvrir et de combattre. Par critique pourtant le $\mathrm{XVII}^{\mathrm{e}}$ siècle ne désigne pas tant « l'esprit critique » que l'ars critiqua, la critique philologique de la tradition humaniste. C'est en tout cas le sens que Naudé donne au mot au chapitre IV de l'Avis pour dresser une bibliothèque lorsqu'il s'interroge sur la place et sur la valeur à accorder aux manuscrits : 
Finalement, la quatrième et dernière de ces maximes n'a pour but que le choix et triage que l'on doit faire de ces manuscrits, pour s'opposer à cette façon introduite et reçue de beaucoup, par la grande vogue qu'ont maintenant les critiques, qui nous ont appris et accoutumé à faire plus d'état de quelques manuscrits de Virgile, Suétone, Perse, Térence, ou quelques autres d'entre les vieux auteurs, que non pas de ceux des galants hommes qui n'ont jamais été vus ni imprimés. Comme s'il y avait quelque apparence de suivre toujours le caprice ou les imaginations de ces nouveaux censeurs et grammairiens qui emploient inutilement le meilleur de leur âge à forger des conjectures et mendier les corrections du Vatican pour changer, corriger ou suppléer le texte de quelque auteur qui aura, peut-être, déjà consommé le labeur de dix ou douze hommes [...]. Et [...] l'exemple de ces censeurs a été tel, et leur autorité si forte, $[\ldots]$ ils ont tellement $[\ldots]$ ensorcelé le monde à leur recherche, qu'il n'y a qu'eux, aujourd'hui, qui soient en vogue et jugés dignes d'être mis dans les bibliothèques. ${ }^{43}$

La charge à l'encontre des petits maîtres zélés qui vont «mendier» des corrections à la Vaticane en dit long sur l'estime que le théoricien de la bibliothèque universelle porte aux critiques de son époque, «censeurs » et "grammairiens » de profession. Pour Naudé, c'est une évidence, les nouveaux représentants de l'école philologique ne sont que les pâles épigones de leurs prédécesseurs. Incapables d'envisager en diachronie la pensée des savoirs constitués, leur objectif se borne à conserver un patrimoine en le sacralisant. Autant dire que leur action sur l'avancée des connaissances est inutile et nulle.

C'est pourtant à cette pratique ancienne que se range encore en 1708 Jean Le Clerc, grand érudit et grand ennemi de Bayle, en publiant une Ars critiqua. Le Clerc désigne par " critique » la critique matérielle et philologique, ce qui le conduit à postuler une vérité du texte reposant sur une prétendue fidélité à la pensée de l'auteur dans la perspective épiphanique de son «établissement». Définition jugée insuffisante par le Dictionnaire universel français et latin qui précise quelques années plus tard :

M. Le Clerc en a donné une idée imparfaite quand il la définit l'art d'enter dans le sens des anciens auteurs, et de faire un juste discernement de leurs véritables ouvrages. D'autres voudraient qu'on la définît simplement l'art de juger. C'est la véritable notion et la signification propre de son nom. ${ }^{44}$

Le changement est capital : à l'ars critica des philologues humanistes succède une critique nouvelle dont la vocation n'est plus seulement de révéler le texte à sa vérité propre mais d'envisager la vérité du texte au regard d'une histoire qui le détermine, bref d'un ailleurs du texte.

Cette méthode d'investigation et d'interprétation est déjà à l'œuvre chez Naudé. Son projet rendu public par Naudé en 1627 est marqué du sceau de l'élan démiurgique, de la jeunesse, de l'ambition. Un savoir est bien en marche contre les "idoles» qui va nécessairement aboutir à l'édification de la plus grande bibliothèque de tous les temps. Naudé revendique en quelque sorte une forme de pyrrhonisme joyeux. Les conditions qui prévalurent à la rédaction du Dictionnaire de Bayle n'ont rien de commun avec celles qui conspirèrent à l'élaboration de l'Avis pour dresser une bibliothèque. À cinquante ans Bayle est libre mais seul, c'est un homme "particulier» comme on disait au XVII siècle, en marge d'une communauté savante et religieuse à laquelle il a longtemps appartenu. Sous la forme annoncée d'un thesaurus des erreurs, son Dictionnaire témoigne à la fois d'un goût assumé

\footnotetext{
${ }^{43}$ Avis pour dresser une bibliothèque, op. cit., p. 189, 191, 193.

${ }^{44}$ Le Dictionnaire universel français et latin, Paris Trévoux, 1721, 5 vol., t. II, entrée « critique », p. 395.
} 
pour la liberté et d'une forme de désillusion constante. Le philosophe sceptique rencontre sur son chemin la figure d'Antisthène et avec lui la conscience de la réversibilité du monde et de ses valeurs ${ }^{45}$. Par l'écriture, par la lecture et par la méthode critique qu'il applique à luimême, Bayle affronte en quelque sorte à ses propres paradoxes en dissociant, et même parfois en opposant raison et croyance. Si le Dictionnaire historique et critique est un anti-Moreri, il est aussi d'une certaine façon aussi un anti-Bayle. La parole marquetée et disséminée de cet ouvrage dit l'aventure d'une conscience à la fois déniaisée et ballottée par l'expérience inconfortable et parfois douloureuse de la liberté de pensée.

Même s'il entretient avec la pensée libertine des rapports de proximité, Bayle dissimule moins qu'il ne tâtonne, empêché par ses propres contradictions, mais toujours emporté par son désir d'en découdre coûte que coûte avec le mensonge, dût-il, ce mensonge, être même parfois le sien ${ }^{46}$. La critique philosophique qu'il applique à la pensée des hommes et qu'il s'applique à lui-même en tant qu'homme de foi doué de raison, le conduit moins, comme Naudé et Bacon, à s'engager dans une logique de progression, qu'à se cantonner avec toujours plus de détermination et d'obstination dans l'aventure de la réflexion.

Aussi Bayle fait-il œuvre de moraliste. Son évaluation critique de la pensée, la chasse aux erreurs à laquelle il soumet inlassablement l'Histoire, le conduit à sonder les arcanes de la nature humaine, et ce faisant à chercher à se connaître dans le monde : c'est à partir de sa propre expérience de la souffrance, par exemple, qu'il en vient à penser le mal ${ }^{47}$. À bien des égards, son Dictionnaire démarque les Essais des Montaigne, ne serait-ce que dans la conception d'une œuvre conçue comme « une perpétuelle pierre d'attente, attente de glose, attente de complément, attente de spécification et de (ré-)interprétation ${ }^{48} »$. Plus ou moins exilé d'une République des lettres dont il pressent la disparition, Bayle s'adresse à un public élargi, qui comprend ceux que d'aucuns nomment alors les «ignorants ». Contrairement à Naudé qui se montre sur ce point intraitable, il légitime la fiction, à la fois pour le plaisir personnel qu'elle lui procure et pour l'éclairage qu'elle lui offre sur lui-même.

Il serait intéressant, à n'en pas douter, d'apprécier les raisons qui justifièrent cette intrusion dans le Dictionnaire historique et critique. Procède-t-elle de la rencontre personnelle avec Pierre-Daniel Huet, théoricien du roman attaché à penser le récit de fiction au sein même des savoirs en lui assignant une vocation éducative ${ }^{49}$ ? Procède-t-elle de l'intérêt progressif d'un savant pour le style, et malgré qu'il en ait pour son propre style ${ }^{50}$, pour cette manière d'écrire non pas dissimulée, cryptée et destinée à un tout petit nombre, mais simplement voilée par une parole ironique et distanciée à même d'être entendue par un public avisé ?

Entre l'idée de bibliothèque théorisée par Naudé en 1627 et la bibliothèque que reflète le Dictionnaire de Bayle l'écart se creuse en tout cas sur ce point. Naudé ferme ses portes aux faibles esprits qui «s'amusent après les fictions et romans ${ }^{51}$ " et ne se résout pas à ce que l'honnête lecteur puisse «se repaitre de telles fables ${ }^{52}$ ». Sa bibliothèque est évidemment

\footnotetext{
${ }^{45}$ Voir Peter Sloterdijk, Critique de la raison cynique, trad. fr. par Hans Hildenbrand, Paris, Christian Bourgois, 2000.

${ }^{46}$ Nous ne suivrons pas sur ce point Sophie Gouverneur pour qui la pensée de Bayle procède très largement d'une écriture de la dissimulation : «Bayle et l'écriture de Naudé », dans Pierre Bayle dans la République des lettres, Paris, Honoré Champion, 2004, études réunies par Antony McKenna et Gianni Paganini, p. 265-286.

${ }^{47}$ Jean-Pierre Jossua, Pierre Bayle ou l'obsession du mal, Paris, Aubier-Montaigne, 1977, p. 166.

${ }^{48}$ J. Lecointe, op. cit., p. 711.

${ }^{49}$ Pierre Bayle témoin et conscience de son temps. Un choix d'articles du Dictionnaire historique et critique, Antony McKenna (dir.), Paris, Honoré Champion, 2001, « Introduction » p. 9.

${ }_{50}$ «L'oserai-je confesser? Le style est une autre cause de ma lenteur : il est assez négligé, il n'est pas exempt de termes impropres, et qui vieillissent, ni peut-être même de barbarismes, je l'avoue, je suis là dessus presque sans scrupules. Mais en récompense je suis scrupuleux jusqu'à la superstition sur d'autres choses plus fatigantes », Dictionnaire historique et critique, par Monsieur Bayle, Rotterdam, Reinier Leers, 1697, 2 vol., t. I, p. 4.

${ }_{51}$ Avis pour dresser une bibliothèque, op. cit., p. 143.

${ }^{52}$ Ibid., p. 151.
} 
interdite aux femmes. Bayle, en revanche, demeure étranger à ces formes de radicalités. Il offre une entrée à Louise Labé dans son ouvrage, par exemple, citant ses œuvres et indiquant leur année d'édition. Mais la plus grande nouveauté tient surtout au fait que l'auteur du Dictionnaire historique et critique envisage clairement la question de la spécificité littéraire. Voici un extrait du très long article qu'il consacre à Marot :

On peut dire sans le flatter non seulement que la poésie française n'avait jamais paru avec les charmes et avec les beautés naturelles dont il l'orna, mais aussi que dans toute la suite du $\mathrm{XVI}^{\mathrm{e}}$ siècle, il ne parut rien qui approchât de l'heureux génie et des agréments naïfs, et du sel de ses ouvrages. Les poètes de La Pléiade sont de fer en comparaison de celui-là, et si au siècle suivant un Voiture, un Sarrazin, un Benserade, et quelques autres l'ont surpassé, ce n'est qu'à cause qu'ils ont trouvé tout fait l'établissement du meilleur goût, et d'une plus grande délicatesse de leur langue. L'incomparable La Fontaine qui s'est reconnu son disciple a contribué beaucoup à remettre en vogue les vers de cet ancien poète. Une infinité de curieux cherchaient ses œuvres avec ardeur, et avaient bien de la peine à les trouver. C'est ce qui a obligé un libraire de La Haye à les remettre sous la presse. Cette édition est très belle. Vous verrez que dans les jugements qu'on a recueillis sur Clément Marot, que les poètes français lui sont redevables du rondeau et qu'ils lui doivent en quelque façon la forme moderne ou le rétablissement du sonnet et du madrigal, et de quelques autres espèces de petits vers. On peut ajouter qu'il fut l'inventeur du mélange des rimes masculines et féminines, qui est une chose sans laquelle notre poésie serait très rude et choquante. Il n'y a que trop de pièces obscènes parmi ses œuvres, et cela fournit un juste sujet de le blâmer. ${ }^{53}$

Bayle découvre de nouvelles catégories, expérimente des «formes inoffensives et non criminelles de divertissement, comme le loisir, [...] la curiosité intellectuelle ${ }^{54} \gg$ ou bien encore le goût de la rareté ${ }^{55}$, s'appliquant à une forme de critique littéraire qui, insensiblement, le conduit à reconsidérer les présupposés d'une anthropologie augustinienne fondée sur la primauté de l'intérêt et des passions. Autant dire que la fiction et son esthétique participent pour Bayle d'un savoir à part entière, non pas certes d'un savoir abstrait et théorique mais d'une forme de connaissance culturelle et existentielle.

Le Dictionnaire historique et critique de Bayle n'accomplit donc pas exactement la bibliothèque universelle théorisée par Naudé dans son Avis pour dresser une bibliothèque. Il ne réalise pas davantage le vaste programme d'histoire des lettres - historia litteraria annoncé avec fracas à la fin du traité paru en 1627 sous le titre de Bibliotheca Memmiana. Certes les deux projets se rejoignent. D'abord sur une méthode consistant à débusquer le mensonge et à promouvoir la vérité dans l'optique non pas d'une classification mais d'une distribution des savoirs ${ }^{56}$. Ensuite sur un objectif commun visant à repenser l'idée d'autorité :

\footnotetext{
${ }^{53}$ Dictionnaire historique et critique par Monsieur Bayle, éd. 1702, op. cit., t. II, p. 2072-2073.

${ }^{54}$ Isabelle Delpha et Philippe de Robert La Raison corrosive, « Les métaphores de la critique », dans Études sur la pensée critique de Pierre Bayle, textes réunis par I. Delpha et Ph. de Robert, Paris, Honoré Champion, 2003, p. 10.

${ }^{55}$ Voir Jean Viardot : « Naissance de la bibliophilie : les cabinets de livres rares », dans Les Bibliothèques sous l'Ancien Régime, 1530-1789, Claude Jolly (dir.), Paris, Promodis-Éditions du Cercle de la Librairie, 1988, p. 269-289; «Livres rares et pratiques bibliophiliques », dans Histoire de l'édition française, Roger Chartier et H.-J. Martin (dir.), t. II, Le Livre triomphant : 1660-1830, Paris, Promodis, 1984, p. 446-467 ; « La Bruyère et le collectionnisme : vraie dévotion à de fausses idoles », Paris, Bibliothèque nationale de France, "Conférences Léopold Delisle », à paraître.

${ }^{56}$ M. Le Dœuff, texte cité, p. XXX.
} 
pour Naudé comme pour Bayle, l'autorité s'évalue et s'apprécie plus qu'elle ne se décrète ou ne s'enregistre.

Reste que Bayle n'est pas installé dans la bibliothèque universelle de Naudé dont il poursuivrait l'entreprise critique en tournant les pages de ses livres innombrables. Quand l'un, " puritain », c'est-à-dire selon le mot de Guy Patin, "fort épuré des sentiments ordinaires ${ }^{57}$ ", $s$ 'efforce de $\left\langle\right.$ tracer le chemin en courant ${ }^{58}$ » en participant sur le mode de l'élan au progrès de la vérité, l'autre, qui « répugne à la ligne droite dans l'écriture ${ }^{59}$ ", en apprécie l'avancée avec suspicion. Quand l'un est héroïque l'autre est distant, ironique, insaisissable et incertain $^{60}$. Quand l'un demeure confiant dans les capacités de l'esprit humain à vaincre l'erreur, l'autre souscrit à l'idée d'une raison non plus progressive mais régressive, d'une « raison corrosive" en somme, au point de n'exclure aucune des voies d'accès à la connaissance, fût-ce celle du mystère :

[...] notre raison n'est propre qu'à brouiller tout et qu'à faire douter de tout : elle n'a pas plutôt bâti un ouvrage qu'elle vous montre les moyens de le ruiner. C'est une véritable Pénélope qui pendant la nuit défait la toile qu'elle avait faite le jour. Ainsi le meilleur usage que l'on puisse faire des études de philosophie est de connaître qu'elle est une voie d'égarement et que nous devons chercher un autre guide qui est la lumière révélée. ${ }^{61}$

\footnotetext{
${ }^{57}$ Citation empruntée à S. Gouverneur : « Bayle et l'écriture de Naudé », texte cité, p. 279.

${ }^{58}$ Avis pour dresser une bibliothèque, op. cit., p. 7.

${ }^{59}$ Hubert Bost, Pierre Bayle historien, critique et moraliste, Turnhout, Brepols, 2006, p. 6.

${ }^{60}$ Voir Pierre Rétat, Le Dictionnaire de Bayle et la lutte philosophique au XVIII siècle, Paris, Les Belles Lettres, 1971, p. 473. Le Dictionnaire historique et critique, œuvre insaisissable et inclassable, conduit la critique contemporaine à interroger la personnalité même de son auteur : pour Elisabeth Labrousse, Bayle est un fidéiste sceptique (Pierre Bayle et l'instrument critique, Paris, Pierre Seghers, 1965 ; Notes sur Bayle, Paris, Jean Vrin, 1987 ; Pierre Bayle : hétérodoxie et rigorisme, Paris, Albin Michel, 1996), pour Gianluca Mori un philosophe crypto-athée (Bayle philosophe, Paris, Honoré Champion, 1986).

${ }^{61}$ Dictionnaire de Bayle, article «Bunel», remarque E. Passage cité en exergue dans le collectif La Raison corrosive, op. cit., p. 7.
} 